\title{
Do Phosphatidylinositides Modulate Vertebrate Phototransduction?
}

\author{
Kyle B. Womack, ${ }^{1}$ Sharona E. Gordon, ${ }^{2}$ Feng He, ${ }^{3}$ Theodore G. Wensel, ${ }^{3}$ Chin-Chi Lu, ${ }^{1}$ and \\ Donald W. Hilgemann'1
}

${ }^{1}$ Department of Physiology, University of Texas Southwestern Medical Center at Dallas, Dallas, Texas 75235-9040, 2 Department of Ophthalmology, University of Washington School of Medicine, Seattle, Washington 98195-6485, and ${ }^{3}$ Department of Biochemistry, Baylor College of Medicine, Houston, Texas 77030

Mammalian rod cyclic nucleotide gated (CNG) channels (i.e., $\alpha$ plus $\beta$ subunits) are strongly inhibited by phosphatidylinositol 4,5-bisphosphate $\left(\mathrm{PIP}_{2}\right)$ when they are expressed in Xenopus oocytes and studied in giant membrane patches. Cytoplasmic Mg-ATP inhibits CNG currents similarly, and monoclonal antibodies to $\mathrm{PIP}_{2}$ reverse the effect and hyperactivate currents. When $\alpha$ subunits are expressed alone, $\mathrm{PIP}_{2}$ inhibition is less strong; olfactory CNG channels are not inhibited. In giant patches from rod outer segments, inhibition by $\mathrm{PIP}_{2}$ is intermediate. Other anionic lipids (e.g., phosphatidyl serine and phosphatidic acid), a phosphatidylinositol-specific phospholipase C, and full-length diacylglycerol have stimulatory effects. Although ATP also potently inhibits cGMP-activated currents in rod patches, the following findings indicate that ATP is used to transphosphorylate GMP, generated from cGMP, to GTP. First, a phosphodiesterase (PDE) inhibitor, Zaprinast, blocks inhibition by ATP. Second, inhibition can be rapidly reversed by exoge- nous regulator of G-protein signaling 9, suggesting G-protein activation by ATP. Third, the reversal of ATP effects is greatly slowed when cyclic inosine $5^{\prime}$-monophosphate is used to activate currents, as expected for slow inosine $5^{\prime}$ triphosphate hydrolysis by G-proteins. Still, other results remain suggestive of regulatory roles for $\mathrm{PIP}_{2}$. First, the cGMP concentration producing half-maximal CNG channel activity $\left(K_{1 / 2}\right)$ is decreased by $\mathrm{PIP}_{2}$ antibody in the presence of PDE inhibitors. Second, the activation of PDE activity by several nucleotides, monitored electrophysiologically and biochemically, is reversed by $\mathrm{PIP}_{2}$ antibody. Third, exogenous $\mathrm{PIP}_{2}$ can enhance PDE activation by nucleotides.

Key words: cIMP; cyclic nucleotide gated channels; DAG; giant patch; ITP; photoreceptors; phosphatydilinositides; phototransduction; PLC; PIP ; RGS proteins; rod cells; transphosphorylation
Activation of a phospholipase C (PLC) is an early step in the phototransduction cascade of invertebrates (Ranganathan et al., 1995), although $\mathrm{IP}_{3}$ does not play a second messenger role (Acharya et al., 1997; Chyb et al., 1999). Whether phosphoinositide metabolism plays any role in vertebrate phototransduction is an open question. Lipid kinases and phospholipases are present in photoreceptor outer segments, and some reports suggest that phosphoinositide metabolism changes in response to light (Hayashi and Amakawa, 1985; Ghalayini and Anderson, 1992, 1995). However, other reports are negative (Van Rooijen and Bazan, 1986).

Recently, phosphatidylinositol 4,5-bisphosphate $\left(\mathrm{PIP}_{2}\right)$ has been shown to modulate the function of several ion channels and transporters, independent of $\mathrm{IP}_{3}$, calcium, DAG, and PKC (Hilgemann and Ball, 1996; Huang et al., 1998). Therefore, we have explored possible roles of PIP $_{2}$ in vertebrate phototransduction, primarily using electrophysiological recordings in excised giant patches. Initially, we examined effects of $\mathrm{PIP}_{2}$ on cyclic nucleotide gated $(\mathrm{CNG})$ channels using cloned bovine channels

Received Dec. 2, 1999; revised Feb. 1, 2000; accepted Feb. 7, 2000.

This work was supported in part by an unrestricted grant from Research to Prevent Blindness (New York, NY) (S.E.G.), National Institutes of Health Grants HL51323 (D.W.H.), EY12374 (S.E.G.), EY07981 (T.G.W.), and EY11900 (T.G.W.), and National Institutes of Health Division of Cell and Molecular Biology Training Program Grant P32 G M 08203 (K.B.W.). We thank Anita Zimmerman for the loan of the night vision equipment.

Correspondence should be addressed to Donald W. Hilgemann, University of Texas Southwestern K4.103, Dallas, TX 75235-9040. E-mail: hilgeman@utsw. swmed.edu.

Copyright (C) 2000 Society for Neuroscience $\quad 0270-6474 / 00 / 202792-08 \$ 15.00 / 0$ expressed in Xenopus oocytes. These effects were evaluated in both $\alpha$ homotetramers and in $\alpha \beta$ heterotetramers. In addition, we studied possible effects of PIP $_{2}$ generated in the oocyte patches in dependence on MgATP. After describing the effects of $\mathrm{PIP}_{2}$ and MgATP on expressed channels, we turn to effects of $\mathrm{PIP}_{2}$ and MgATP in photoreceptor outer segments. Giant rod cell patches allow us to examine regulation of the phototransduction cascade while maintaining cytoplasmic access. Our results demonstrate remarkably efficient, membrane-tethered guanine nucleotide phosphorylation mechanisms in excised patches. Furthermore, our results suggest that phosphoinositides promote the activation of phosphodiesterase (PDE) by transducin, as well as modulate CNG channel activity. Our conclusions about PDE activation are supported by both electrophysiological and biochemical measurements.

\section{MATERIALS AND METHODS}

Expression of cloned channels. The cDNA clone of the $\alpha$ subunits (Kaupp et al., 1989) was provided by W. N. Zagotta (University of Washington, Seattle, WA) and the cDNA clone of the $\beta$ subunits (Chen et al., 1993) was supplied by K. W. Yau (Johns Hopkins University, Baltimore, MD). The cDNAs were both in a pGMEHE vector supplied by E. R. Liman (University of Southern California, Los Angeles, CA). RNA was transcribed using the Mmessage Mmachine in vitro transcription kit (Ambion, Austin, TX) and was injected into Xenopus oocytes, which were maintained at $14^{\circ} \mathrm{C}$ for $3-10 \mathrm{~d}$.

Isolation of photoreceptor cells. All results presented from photoreceptor cells are patch-clamp records from Xenopus rod cells. The frogs were commercially obtained and kept at room temperature with $12 \mathrm{hr}$ light/ dark cycles, except when the experiment required dark adaptation. The frogs were decapitated and double-pithed. The eyes were quickly re- 
moved, and the globe was sectioned in half and placed immediately in a modified frog Ringer's solution at $4^{\circ} \mathrm{C}$, which consisted of $\mathrm{NaCl} 111 \mathrm{~mm}$, $\mathrm{KCl} 2.5 \mathrm{~mm}, \mathrm{CaCl}_{2} 1 \mathrm{~mm}$, HEPES $3 \mathrm{~mm}, \mathrm{MgCl}_{2} 1.2 \mathrm{~mm}$, dextrose $10 \mathrm{~mm}$, and EDTA $20 \mu \mathrm{M}$, pH 7.6 with $N$-methyl-glucamine (NMG). Retinas were then removed under a dissecting microscope and stored in the same modified frog ringer solution at $4^{\circ} \mathrm{C}$. Dissociated rod outer segments were obtained by gentle agitation of the pieces of retina just before each experiment. As noted in Results, we have performed similar experiments with rod cells from salamanders, cone cells from catfish, and rod cells from mice.

Electrophysiological recordings. Recordings were made from excised, giant patches in the inside-out configuration as described previously (Hilgemann and $\mathrm{Lu}, 1998$ ). Pipette tip diameters were 15-40 $\mu \mathrm{m}$ for oocyte patches and were $\sim 5-7 \mu \mathrm{m}$ for rod cell patches. In the rod cell recordings, the diameter of the pipettes was large enough, relative to the diameter of the rod cells, so that the cells generally doubled over and were squeezed into the pipette tip by $5-10 \mu \mathrm{m}$ before the gigaohm seal formed. The two protruding ends of the rod cell were then broken off with a solution stream from a polyethylene tube pointed at the patch tip. This technique allowed retention of $\sim 3-50 \%$ of the cell in the patch. Most recordings were made at a holding potential of $0 \mathrm{mV}$ using a pipette solution containing (in mM) $\mathrm{KCl} 20$, $\mathrm{HEPES} \mathrm{10,} \mathrm{CaCl}_{2}$ 2, and NMG 80, $\mathrm{pH} 7.0$, and a bath solution containing $\mathrm{KCl} 90$, EGTA 2, $\mathrm{MgCl}_{2} 0.5$, and HEPES 10. Data shown for currents recorded at $+100 \mathrm{mV}$ used symmetrical solutions consisting of $\mathrm{NaCl} 130 \mathrm{~mm}$, HEPES $3 \mathrm{~mm}$, and EDTA $200 \mu \mathrm{M}, \mathrm{pH}$ 7.2. Recordings were made with either an Axopatch 1D or an Axopatch 200 patch-clamp amplifier (Axon Instruments, Foster City, CA). Data were filtered at $2 \mathrm{kHz}$, and either an ITC-18 computer interface with Pulse data acquisition software (Instrutech, Port Washington, NY) or a DigiData 1200 (Axon Instruments) with our own software was used for voltage protocols and digital data acquisition. The long-time current records presented are strip chart recordings (Kipp and Zonen, Suskatoon, Saskatchewan, Canada). In all results presented, we define the CNG channel-mediated current as that current activated by 8-bromo-cGMP, cGMP, or cyclic inosine 5'-monophosphate (cIMP). Initially, we performed experiments with 8-bromo-cGMP under the assumption that this analog would not be cleaved by rod PDE. However, we determined that phosphodiesterase inhibitors could reverse effects of triphosphonucleotides in rod patches, whether submaximal concentrations of 8-bromo-cGMP (5-10 $\mu \mathrm{M})$ or cGMP (0.1-1 mM) were used. We assume that this difference in concentration reflects approximately the relative ability of rod PDE to cleave these two nucleotides. In this light, we present results with both cyclic nucleotides.

Biochemistry. Rod outer segments were prepared from bovine retinas as described previously (Papermaster and Dreyer, 1974), and cGMP phosphodiesterase activity was assayed using the $\mathrm{pH}$ recording method (Liebman and Evanczuk, 1982; Malinski and Wensel, 1992). Recombinant $\mathrm{His}_{6}$-RGS9dc (RGS, regulator of G-protein signaling) containing residues 291-484 of RGS9-1 (pET14b), His $_{6}-\mathrm{PDE} \gamma$ (pET14b), and His $_{6}$-RGS16 (pET15b) were expressed and purified by standard proce-

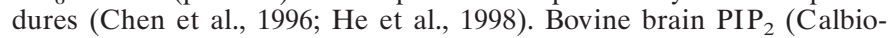
chem, La Jolla, CA) was dried under a stream of nitrogen and dissolved in Milli-Q water by sonication. $\mathrm{PIP}_{2}$ antibody $(\mathrm{Ab})$ (PerSeptive Biosystems, Foster City, CA) supplied in calf serum was used at a 1:20 dilution. As controls, mouse $\mathrm{IgG}_{2 \mathrm{~B}}$ and fetal bovine serum (FBS) was used at the same total protein concentration.

\section{RESULTS}

Rod CNG channels, when expressed in Xenopus oocytes and studied in conventional excised patches, are inhibited by application of MgATP to the cytoplasmic membrane face, and this effect of ATP can be blocked by inhibitors of tyrosine kinases (Molokanova et al., 1997). We have described previously that the same protocols in oocyte patches result in the phosphorylation of phosphatidylinositol to generate PIP $_{2}$, which activates a number of ion channels and $\mathrm{Na} / \mathrm{Ca}$ exchangers (Hilgemann and Ball, 1996; Huang et al., 1998). Therefore, we tested whether phosphatidylinositides might modulate $\mathrm{CNG}$ channel function in giant excised patches from oocytes, and Figure 1 describes our typical results.

Bovine CNG channels ( $\alpha$ and $\beta$ subunits) were expressed in Xenopus oocytes, and current was defined in giant inside-out

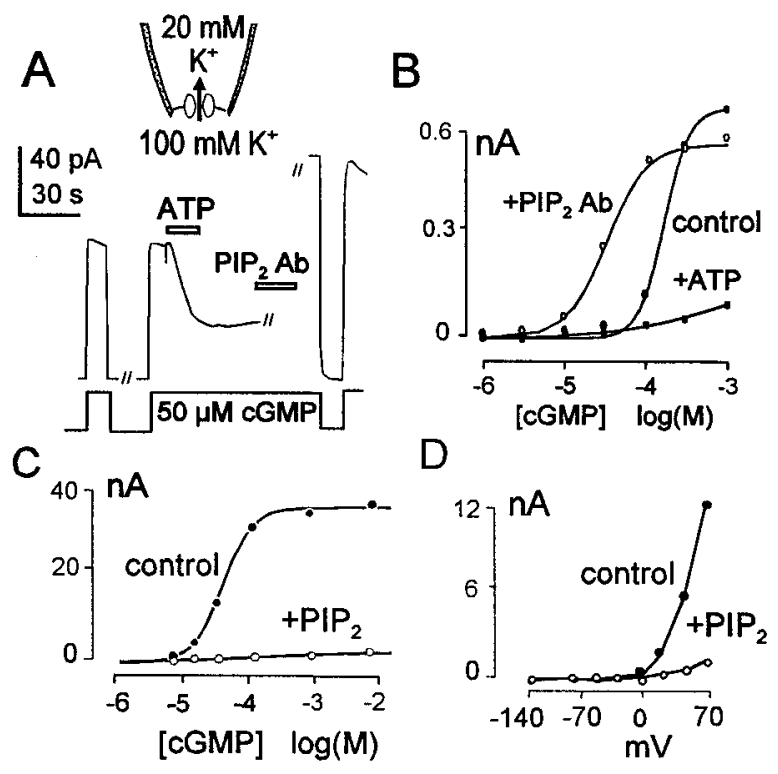

Figure 1. Effects of ATP, $\mathrm{PIP}_{2}$, and $\mathrm{PIP}_{2} \mathrm{Ab}$ on the current through rod CNG channels ( $\alpha$ plus $\beta$ subunits) expressed in oocytes and monitored in excised giant patches. $A$, Inhibitory effects of $1 \mathrm{~mm} \mathrm{MgATP}$ applied in the presence of $50 \mu \mathrm{M}$ cGMP with reversal by $\mathrm{PIP}_{2} \mathrm{Ab}$. B. Effect of ATP (ם) and $\mathrm{PIP}_{2} \mathrm{Ab}(\mathrm{O})$ on cGMP dependence of the $\mathrm{CNG}$ channel current compared with control $(\bullet)$. $C$, Effect of PIP $P_{2}(\bigcirc)$ on cGMP dependence of $\mathrm{CNG}$ current compared with control $(\bullet)$. PIP $_{2}$ was applied as liposomes in a bulk concentration of $30 \mu \mathrm{M}$. $D, I-V$ responses of the CNG channels before $(\bullet)$ and after $(\bigcirc) \mathrm{PIP}_{2}$ was applied with cGMP at $50 \mu \mathrm{M}$. The outward rectification is from block of the channels by $\mathrm{Ca}^{2+}$ in the pipette.

patches by application and withdrawal of cGMP in the presence of an outwardly directed potassium gradient. Each panel of Figure 1 shows data from a different patch.

As shown in Figure $1 A$, application of $\operatorname{MgATP}(2 \mathrm{~mm})$ in the presence of $50 \mu \mathrm{M}$ cGMP resulted in a strong inhibition of the cGMP-dependent current over the course of 20-60 sec, and current remained inhibited after ATP was withdrawn. Subsequent application of a $\mathrm{PIP}_{2}$ antibody, shown to be effective in previous studies (Huang et al., 1998), completely reversed this inhibitory effect and elevated the current above its baseline level but still retained its cGMP dependence (Fig. $1 A$ ). Figure $1 B$ shows how ATP and the PIP $_{2}$ antibody affect the cGMP dependence of the CNG channel current at $0 \mathrm{mV}$. The inhibitory effect of ATP is so strong that the cGMP dependence cannot be determined using cGMP concentrations up to $1 \mathrm{mM}$. The subsequent activation of current by $\mathrm{PIP}_{2} \mathrm{Ab}$ shifts the cGMP dependence of current to a lower concentration range $\left(K_{1 / 2}, 25 \mu \mathrm{M}\right)$ than for control current $\left(K_{1 / 2}, 130 \mu \mathrm{M}\right)$, in addition to restoring the maximum current to near the control level (Fig. $1 B$ ). As shown in Figure $1 C$, application of $\mathrm{PIP}_{2}$ liposomes $(30 \mu \mathrm{M})$ inhibited the cGMP-activated currents even more potently than ATP. The typical outwardly rectifying current-voltage relationships of the cGMP-activated current were simply scaled down by the lipid (Fig. 1D). The PDE inhibitors Zaprinast and IBMX had no effect on the actions of either ATP or $\mathrm{PIP}_{2}$ in the oocyte patches (results not shown).

To gain further insight into the mechanism of $\mathrm{PIP}_{2}$ in the expressed channels, we examined the limited effects of brief $\mathrm{PIP}_{2}$ applications. Also, we examined the effects of $\mathrm{PIP}_{2}$ when only the $\alpha$ subunit of CNG channels was expressed, and we examined the 

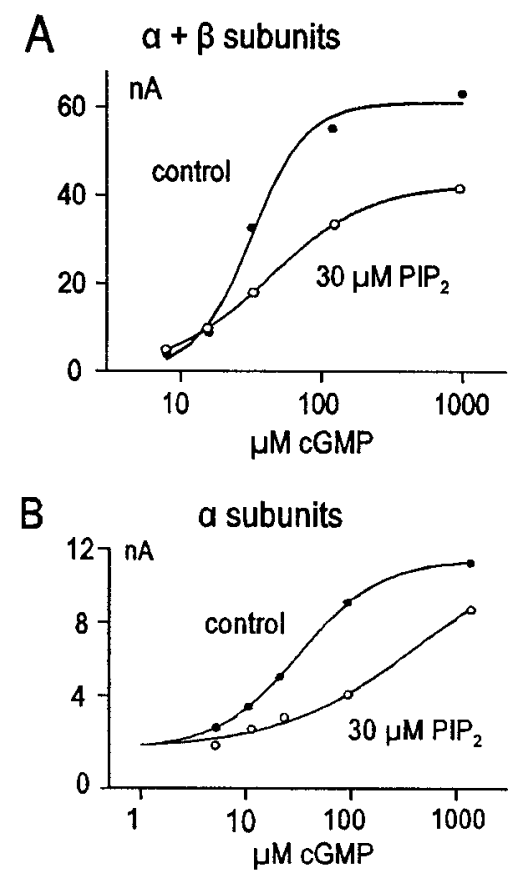

Figure 2. Different effects of $\mathrm{PIP}_{2}$ on $\alpha \beta$ heterotetrameric channels and $\alpha$ homotetrameric channels. $A$, Effect of $\mathrm{PIP}_{2}(\bigcirc)$ compared with control (๑) on cGMP dependence of current through $\alpha \beta$ heterotetrameric CNG channels. Note the decrease in maximum current without appreciable change of $K_{1 / 2}$. B, Effect of PIP $(\bigcirc)$ compared with control $(\bullet)$ on cGMP dependence of current through $\alpha$ homotetrameric channels. Note the relative lack of effect on the maximum current (still climbing at the highest cGMP concentration) with a shift to the right of the apparent $K_{1 / 2}$.

effects of other anionic lipids. As shown in Figure $2 A$, brief application of $\mathrm{PIP}_{2}$ (or application of low $\mathrm{PIP}_{2}$ concentrations) resulted in a clear reduction of the maximum current and sometimes resulted in a small increase of currents with the lowest cGMP concentrations. Thus, the slope of the concentrationcurrent relationship typically decreased. Two differences were striking when only the $\alpha$ subunit was expressed (Fig. 2B). First, the maximal inhibitory effects of $\mathrm{PIP}_{2}$ obtained were much smaller. Second, $\mathrm{PIP}_{2}$ acted only to shift the concentrationcurrent relationship to higher cGMP concentrations with no effect on the maximal current. Clearly, a major component of the $\mathrm{PIP}_{2}$ effect requires the presence of the $\beta$ subunits of the channels. We mention that we also examined $\mathrm{PIP}_{2}$ effects on olfactory CNG channels expressed in oocyte patches, and either no effect or a modest stimulatory effect was observed. In results not shown, we found that phosphatidyl serine (PS) and phosphatidic acid (PA) both stimulated CNG currents in channels composed of both $\alpha$ and $\beta$ subunits. All of these results suggest that the effects of $\mathrm{PIP}_{2}$ in the rod CNG channels are rather specific and could be of physiological importance.

To investigate the possible physiological relevance of the findings using oocyte expression, we performed related experiments in giant excised patches from amphibian (Xenopus) rod photoreceptor outer segments that included disk membranes. When cGMP-activated currents were recorded in such patches, application of either MgATP or MgGTP resulted in prompt inhibition of CNG current (Fig. 3). As illustrated by the results in Figure 3, very strong current inhibition was also obtained when the hydrolysis-resistant cGMP analog 8-bromo-cGMP was used at an approximately half-maximal concentration $(10 \mu \mathrm{M})$. After nucle-
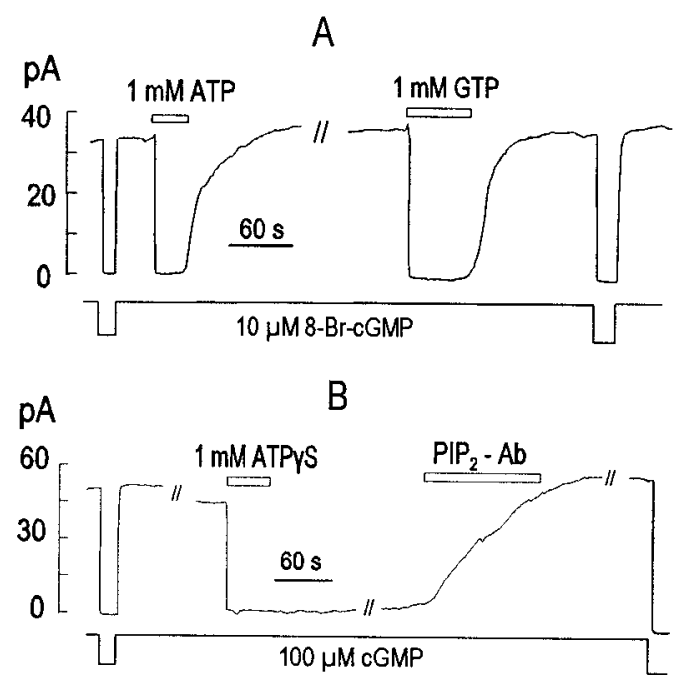

Figure 3. Effects of ATP, GTP, and ATP- $\gamma-\mathrm{S}$ on CNG current from excised giant patches of amphibian rod outer segments. $A$, Reversible inhibition of current by ATP and GTP in the presence of 8-bromo-cGMP $10 \mu$ M. $B$, Long-term inhibition of current by ATP- $\gamma$-S with reversal by $\mathrm{PIP}_{2} \mathrm{Ab}$.

A $\quad B$
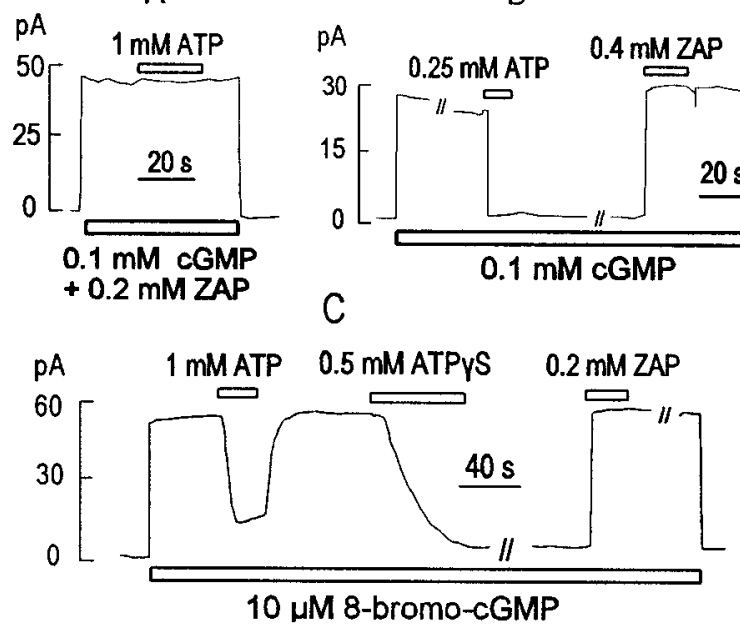

Figure 4. ATP and ATP- $\gamma$-S effects on amphibian rod cells are mediated by activation of PDE. $A$, Zaprinast $(200 \mu \mathrm{M})$, a potent inhibitor of PDE, blocks the ATP effect in rod outer segments. $B$, ATP would sometimes have a long-lasting inhibitory effect on rod outer segment current at low to moderate cGMP concentrations $(<200 \mu \mathrm{M})$. Zaprinast $(200 \mu \mathrm{M})$ reverses this long-lasting ATP-induced inhibition. $C$, Zaprinast $(200 \mu \mathrm{M})$ reverses long-lasting inhibitory effects of ATP- $\gamma-\mathrm{S}$.

otide washout, in the presence of half-maximal or higher cyclic nucleotide concentrations, the effects of ATP and GTP typically reversed with very similar time courses after a short delay. With lower cyclic nucleotide concentrations, the inhibitory effect of ATP often did not reverse after washout (Fig. 4). As shown in Figure $3 B$, the ATP analog ATP- $\gamma$-S typically inhibited the current equally or more strongly than ATP. This inhibitory effect could never be washed out, but the control current amplitudes could usually be restored entirely by application of the PIP Ab. In most cases $(>10)$, current could be inhibited by ATP- $\gamma-\mathrm{S}$ and rescued with PIP Ab multiple times in the same patch.

Although these effects of nucleotides in rod patches appear similar to those observed in oocyte patches, the underlying mech- 


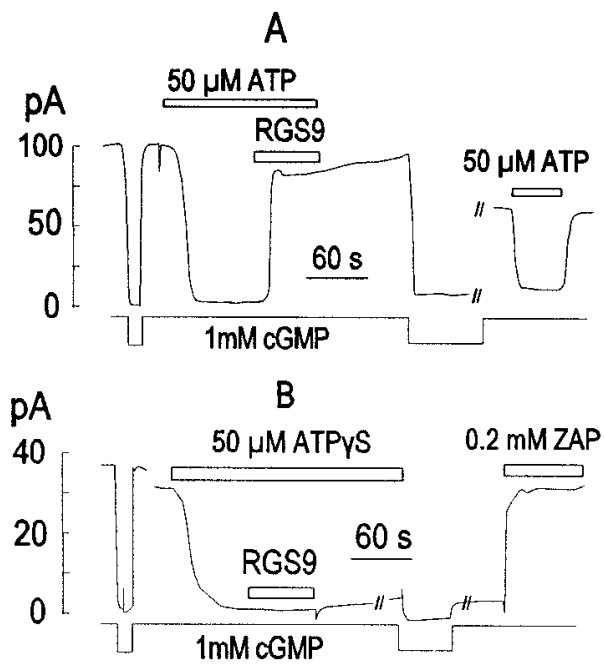

Figure 5. RGS9 antagonizes ATP effects in rod outer segments consistent with its known GAP activity. $A$, RGS9 $(2.4 \mu \mathrm{M})$ reverses the inhibition of current by simultaneously applied $50 \mu \mathrm{M}$ ATP. $B$, RGS9 does not reverse the inhibition of current by ATP- $\gamma-\mathrm{S}$.

anisms are clearly very different. As shown in Figure $4 A$ and in contrast to results in oocyte patches, nucleotides were usually without effect in rod patches when experiments were performed in the presence of a high concentration $(0.2 \mathrm{~mm})$ of the PDE inhibitor Zaprinast or IBMX (2 mM; data not shown). As shown in Figure $4 B$, we sometimes observed that inhibitory effects of ATP did not reverse after removal of ATP, a pattern very similar to oocyte results. However, these long-term effects were also reversed by Zaprinast application. Figure $4 C$ shows the typical fast reversal by Zaprinast of the long-lasting inhibition of CNG current induced by ATP- $\gamma$-S application. Thus, these results are mostly consistent with the nucleotides activating PDE as suggested previously (Ertel, 1994). One observation, which remains enigmatic, is that current often remains high after extensive washout of Zaprinast (Fig. 4B,C). In extensions of this protocol (data not shown), current could be inhibited and reactivated multiple times by brief application of ATP- $\gamma-\mathrm{S}$ (or ATP) and subsequent brief reapplication of Zaprinast.

How is PDE activated by ATP and ATP- $\gamma$-S? The phosphorylation of lipids or proteins seemed unlikely, because several specific and nonspecific phosphatase inhibitors had no effect on the reversal of these ATP effects (data not shown). The explanation, favored by experiments described subsequently, is that GTP and thio-phosphorylated guanine triphosphates are generated in the patches from their precursors via powerful transphosphorylation reactions (Swarup and Garbors, 1983; Yuen et al., 1989; Otero, 1990; Piacentini and Niroomand, 1996) with subsequent activation of transducin and therefore PDE. Our first evidence, described in Figure $5 A$, is that the effect of low concentrations of ATP can be reversed (or antagonized) quickly by an intervention known to increase GTPase activity of the $\mathrm{T} \alpha$ subunit of transducin.

In general, RGS proteins exert a GTPase-activating protein (GAP)-like action in G-protein signaling (Berman and Gilman, 1998), and the RGS9 protein, in particular, is thought to exert this function in photoreceptors (He et al., 1998). As indicated in Figure $5 A$, the $C N G$ current was first inhibited by applying a low ATP concentration $(50 \mu \mathrm{M})$. Then, in the continued presence of ATP, the RGS9 catalytic subunit $(2.4 \mu \mathrm{M})$ was applied, and the

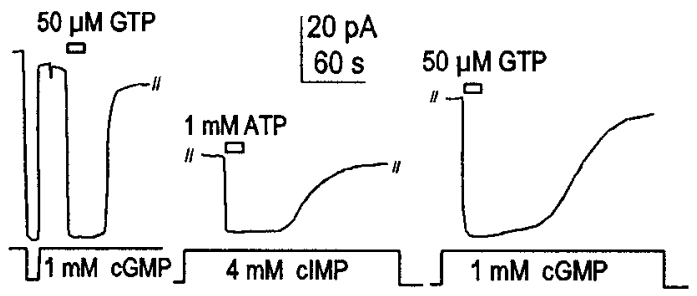

Figure 6. GTP and ATP are used as substrates by guanylate kinase and NDK to produce GTP and ITP from hydrolyzed cGMP and cIMP, respectively. Both GTP and ITP readily activate G-proteins, including transducin, but ITP is hydrolyzed to IDP by the G $\alpha$ subunit much more slowly than GTP is hydrolyzed to GDP (Klinker and Seifert, 1997).

current was rapidly returned to nearly its control amplitude. As would be expected for an acceleration of the G-protein cycle, the RGS protein was not effective in reversing effects of high ATP concentrations (data not shown). A further prediction was that the RGS protein would not reverse the effect of ATP- $\gamma$-S, because the resulting GTP- $\gamma$-S would be resistant to hydrolysis in any circumstance. As shown in Figure 5B, the RGS9 was without effect on the inhibitory effect of $50 \mu \mathrm{M}$ ATP- $\gamma$-S.

A second type of evidence for transphosphorylation, described in Figure 6, is that the types of phosphonucleotides present in the experiment can strongly affect the reversal rates of the ATP (and GTP) effects. The example we choose to present is the effect of using cIMP, instead of cGMP, to activate the CNG channels; cIMP activates rod CNG channels with an effectiveness intermediate between cAMP and cGMP (Tanaka et al., 1989; Varnum et al., 1995). It is known that IMP can be phosphorylated by nucleotide kinases, including guanylate kinase, although at a much slower rate (Yan and Tsai, 1999). Nucleotide diphosphate kinases (NDKs), including one isolated from bovine retina, are known to have "broad specificity for the nucleotide substrate" (Abdulaev et al., 1998), and inosine 5'-triphosphate (ITP) can substitute for GTP in most G-proteins, including transducin. However, ITP is hydrolyzed to inosine $5^{\prime}$-diphosphate (IDP) by the T $\alpha$ subunit much more slowly than GTP (Kelleher et al., 1986; Klinker and Seifert, 1997). Thus, it should be possible to monitor current with cIMP and generate ITP from ATP $($ cIMP $\rightarrow$ IMP $\rightarrow$ IDP $\rightarrow$ ITP). If the ATP effect is produced by transphosphorylation of IDP, there should be a distinct change in the reversal kinetics of the ATP effect when using cIMP to activate current (Yee and Liebman, 1978), assuming that reversal reflects phosphonucleotide hydrolysis by the G-protein. We have verified this prediction repeatedly, and Figure 6 illustrates the results that we consider most important.

In the experiment described in Figure 6, the response to GTP was first examined in the presence of cGMP. Current was completely inhibited, and after GTP removal, the current reactivated quickly to nearly the control level after a delay of $\sim 15 \mathrm{sec}$. From our experience in $>100$ such protocols, the kinetics of the ATP reversal would be nearly identical. Next, in the experiment, we activated current with $4 \mathrm{~mm}$ cIMP and examined the effect of ATP. Reversal of the ATP effect occurred slowly over $>60 \mathrm{sec}$ after a nearly $60 \mathrm{sec}$ delay. As further illustrated in this experiment, the reversal of GTP effects remained more than five times slower than the control GTP response, even when cIMP was removed and cGMP was again used to activate the CNG current. This result implies that ITP can be generated from residual IMP and IDP, bound in the patch, after $>1$ min of washout. Furthermore, the result implies that nucleotides generated from 


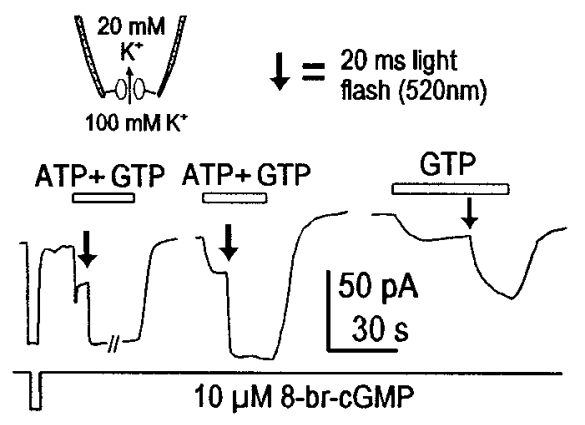

Figure 7. Effect of transphosphorylation on the light response. This is a recording from a dark-adapted amphibian rod, performed in darkness. Current is defined with 8-bromo-cGMP, and light flashes $(20 \mathrm{msec}$ at 520 $\mathrm{nm}$ ) are given either in the presence of $2 \mathrm{mM}$ ATP plus $20 \mu \mathrm{M}$ GTP (1st two light responses) or in the presence of $20 \mu \mathrm{M}$ GTP alone. Note the slower kinetics and smaller magnitude of the GTP-only response.

transphosphorylation reactions are preferentially available to activate the G-protein.

Is transphosphorylation important to understand the kinetics of light responses? To address this question, we examined light responses of dark-adapted rods outer segments in excised giant patches. This is possible because the disk membranes that contain the necessary components of the phototransduction cascade can remain attached to the plasma membrane when a patch has been excised (Ertel, 1990). We first dark-adapted frogs for 6-12 hr. We then harvested retinal tissue in the dark, using night vision goggles and a dim infrared light source. Patches were made under video control using dim $800 \mathrm{~nm}$ light with our usual pipettes. 8-Bromo-cGMP was used to define the current instead of cGMP. Light flashes were delivered at a wavelength of $520 \mathrm{~nm}$ for 20 msec in either the presence of ATP 2 mM plus GTP $20 \mu \mathrm{M}$ or GTP $20 \mu \mathrm{M}$ alone. We observed larger and faster light responses when $2 \mathrm{~mm}$ ATP was present, in addition to $20 \mu \mathrm{M}$ GTP, than when 20 $\mu \mathrm{M}$ GTP was used alone (Fig. 7). Thus, transphosphorylation could influence phototransduction kinetics under physiological conditions.

As outlined up to now, we have obtained no clear evidence that $\mathrm{PIP}_{2}$ is generated in rod patches when ATP is applied in the presence of PDE inhibitors. Next, therefore, we studied the direct effects of $\mathrm{PIP}_{2}$, and other lipids in the native rod membranes. $\mathrm{PIP}_{2}$ liposomes produced inhibition of $\mathrm{CNG}$ current in rod patches, although the effects were usually smaller than in oocyte patches; the result shown in Figure $8 A$ is one of the larger responses obtained. To minimize any effects of PDE activity, we examined the effects of $\mathrm{PIP}_{2}$ (Fig. $8 B$ ) and $\mathrm{PIP}_{2} \mathrm{Ab}$ on the concentration dependence of $\mathrm{CNG}$ current using 8-bromo-cGMP in the presence of $0.2 \mathrm{~mm}$ Zaprinast. The effect of $\mathrm{PIP}_{2}$ is very similar to that described for a brief $\mathrm{PIP}_{2}$ application in oocyte patches with the cloned $\alpha \beta$ heterotetramers (Fig. $2 A$ ), namely a reduction of the maximal current with a small increase of current in the low concentration range. The effect of $\mathrm{PIP}_{2} \mathrm{Ab}$ (Fig. $8 C$ ) is also qualitatively similar to results obtained in oocytes; the halfmaximal nucleotide concentration is shifted to the left, and the slope of the concentration-response relationship is somewhat reduced.

Previously, a short-chain (8:0) DAG analog has been shown to inhibit CNG currents in rod cell patches (Gordon et al., 1995), and therefore $\mathrm{PIP}_{2}$ might possibly act on $\mathrm{CNG}$ channels by providing a source of DAG. To address this possibility, we reexamined how DAG affects CNG currents. First, we confirmed that

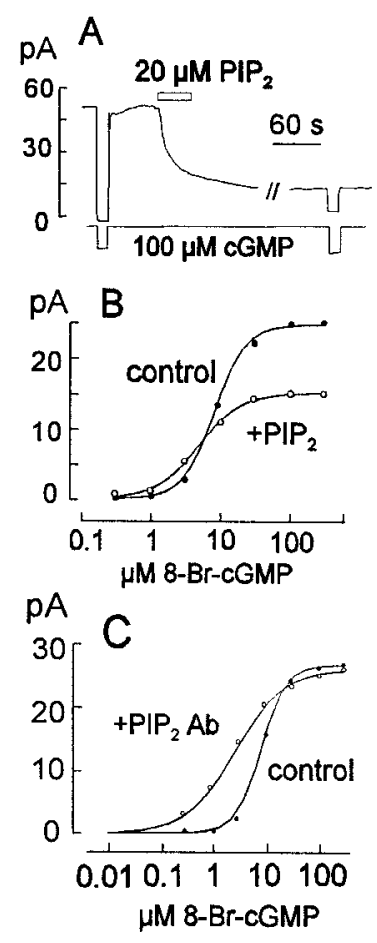

Figure 8. Effects of $\mathrm{PIP}_{2}$ and $\mathrm{PIP}_{2} \mathrm{Ab}$ on $\mathrm{CNG}$ current in rod outer segment giant patches. $A$, Inhibitory effect of $\mathrm{PIP}_{2}$ applied as liposomes in the presence of cGMP. $B$, Effect of $\mathrm{PIP}_{2}(\bigcirc)$ compared with control $(\bullet)$ on the cyclic nucleotide dependence of current from rod outer segments (using 8-bromo-cGMP and $200 \mu \mathrm{M}$ Zaprinast to eliminate any effects through PDE). $C$, Effect of $\mathrm{PIP}_{2} \mathrm{Ab}(\bigcirc)$ compared with control $(\mathbf{O})$ on the cyclic nucleotide dependence of current from rod outer segments (again using 8-bromo-cGMP and $200 \mu \mathrm{M}$ Zaprinast).

short-chain (8:0) DAG did indeed have an inhibitory effect on the rod current (Fig. 9A), namely by reducing the maximal current in response to increasing concentrations of 8-bromo-cGMP. Second, we tested effects of full-length DAG, both DAG generated from endogenous lipids of the patch and DAG added exogenously.

On the basis of previous studies, it seems possible to deplete membranes of phosphatidylinositol by converting it to DAG with a bacterial, phosphatidylinositol-specific PLC (Hilgemann and Ball, 1996). Thus, it is expected that very large DAG concentrations can be generated in native membranes with this approach. However, application of the PLC did not result in current inhibition but rather a modest stimulation, and very similar results were obtained in oocyte patches (data not shown). As shown in Figure $9 B$, the stimulatory effect results in a small leftward shift of the cyclic nucleotide dependence of the channels.

Because DAG alone does not form liposomes, we tested the effects of full-length DAG by incorporating it into liposomes containing other lipids. Briefly, we prepared liposomes containing $10 \%$ dipalmityl-DAG with either $90 \%$ PA or $90 \%$ PS. Patches were first equilibrated with either pure PA or PS vesicles (apparent concentration, $2 \mathrm{~mm}$ ). Then, vesicles containing DAG with the other lipid were applied. As shown in Figure $9 C$, application of PS vesicles resulted in a small stimulation of current, and subsequent application of the PS-DAG mixed vesicles resulted in a small additional stimulation. A similarly small stimulatory effect of DAG was found using PA-DAG vesicles (data not shown). Thus, we conclude that full-length DAG probably does not inhibit rod CNG channels. 

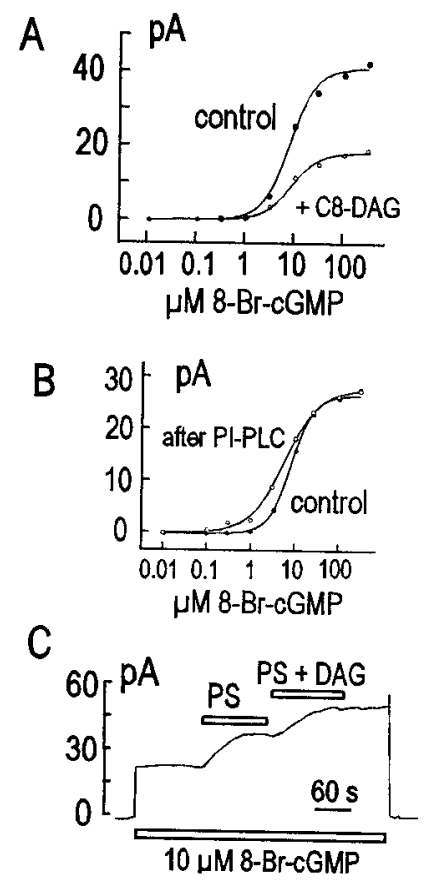

Figure 9. Different effects of short-chain (8:0) DAG and either phosphatidylinositol PLC (PI-PLC) (presumably liberating native DAG) or (16:0) DAG on the rod outer segment CNG current. $A$, Inhibitory effect of short-chain (8:0) DAG $(\bigcirc)$ on the cyclic nucleotide dependence of current compared with control (৩). B, Stimulatory effect of phosphatidylinositol PLC $(0.6 \mathrm{U} / \mathrm{ml})(\bigcirc)$ on the cyclic nucleotide dependence of current compared with control $(\bullet)$. C, Effect of PS applied as liposomes, followed by (16:0) DAG delivered as a $10 \%$ mixture in PS vesicles.

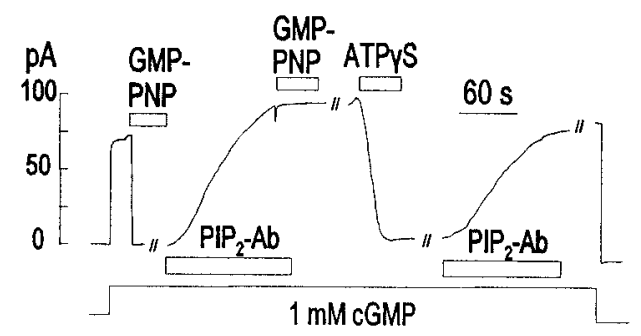

Figure 10. Recording from a rod outer segment giant patch at $0 \mathrm{mV}$ with an outwardly directed $\mathrm{K}^{+}$gradient. GMPPNP activates transducin, which activates PDE. PIP ${ }_{2} \mathrm{Ab}$ reverses the PDE activation, but GMPPNP is not able to have a second effect, although it should still be able to activate transducin. ATP- $\gamma$-S has an effect in addition to activation of transducin through transphosphorylation. PIP $_{2} \mathrm{Ab}$ can then reverse the PDE activation produced by ATP- $\gamma$-S. This effect may involve generation of $\mathrm{PIP}_{2}$ and would imply a requirement for $\mathrm{PIP}_{2}$ to properly activate PDE through transducin.

Finally, we describe results with the $\mathrm{PIP}_{2} \mathrm{Ab}$ that appear important, but which we cannot interpret confidently at this time. As described previously, the $\mathrm{PIP}_{2} \mathrm{Ab}$ can reverse effects of ATP- $\gamma$-S in rod patches (Fig. 3B). Assuming that a thiophosphorylated GTP analog is generated in such experiments, it was a question whether $\mathrm{PIP}_{2} \mathrm{Ab}$ might reverse the activation of PDE by other GTP analogs. The GTP analog GMPPNP can activate G-proteins, including $\mathrm{T} \alpha$, but it cannot be hydrolyzed by the GTPase activity of T $\alpha$ or act as a substrate for kinases. As shown in Figure 10, application of $0.1 \mathrm{~mm}$ GMPPNP results in complete inhibition of the CNG channel currents in rod patches. This effect persists indefinitely after removing the nucleotide

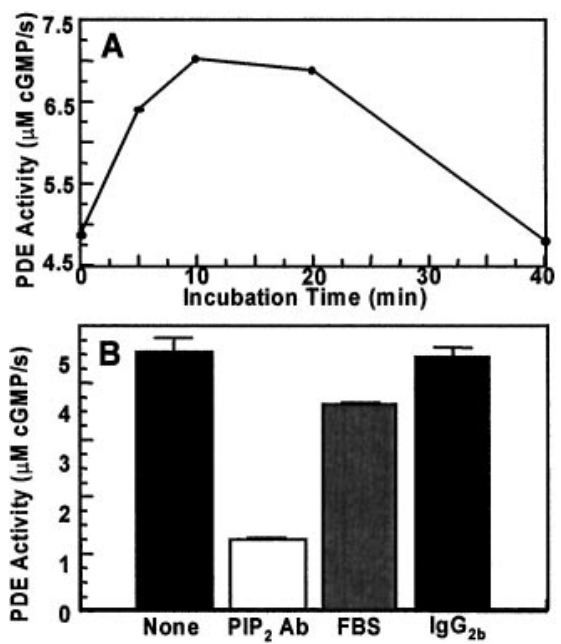

Figure 11. Effects of $\mathrm{PIP}_{2}$ and $\mathrm{PIP}_{2} \mathrm{Ab}$ on G-protein activation of PDE. $A$, Time course of $\mathrm{PIP}_{2}(1.8 \mu \mathrm{M})$ stimulation of PDE activity. $B$, Inhibition of PDE activation by $\mathrm{PIP}_{2} \mathrm{Ab}$. PDE activity was assayed using bovine rod outer segments at $2.5 \mu \mathrm{M}$ rhodopsin, with $2 \mathrm{mM}$ cGMP as substrate. PDE activation was initiated by addition of GMPPNP $(100 \mu \mathrm{M})$. Control treatments with $\mathrm{IgG}_{2 \mathrm{~b}}$ and FBS contained the same total protein concentration $(0.46 \mathrm{mg} / \mathrm{ml})$ as the sample treated with $\mathrm{PIP}_{2} \mathrm{Ab}$. Inhibition by $\mathrm{PIP}_{2} \mathrm{Ab}$ was approximately linear in the concentration range of 1:80-1:20 dilution. $\mathrm{PIP}_{2}$ and $\mathrm{PIP}_{2} \mathrm{Ab}$ did not have detectable effects on either basal PDE activity or PDE activity stimulated by trypsin treatment. Similar effects of $\mathrm{PIP}_{2} \mathrm{Ab}$ were observed using GPP-CH$-\mathrm{CH}_{2}-\mathrm{P}$ or GTP- $\gamma-\mathrm{S}$ to stimulate PDE (data not shown).

(data not shown), but application of the $\mathrm{PIP}_{2} \mathrm{Ab}$ can completely reverse the effect over 2 to $3 \mathrm{~min}$. After removal of the $\mathrm{PIP}_{2} \mathrm{Ab}$, application of GMPPNP is without effect; in some experiments small, reversible effects of GMPPNP were observed. Whereas GMPPNP remains without effect after antibody, ATP- $\gamma-\mathrm{S}$ can again inhibit the current, and the inhibition is reversed by the $\mathrm{PIP}_{2} \mathrm{Ab}$. We note that we obtained similar results using GTP- $\gamma-\mathrm{S}$ instead of GMPPNP (data not shown).

To test our interpretation that these effects reflect changes of PDE activity, we tested for effects of $\mathrm{PIP}_{2}$ and $\mathrm{PIP}_{2} \mathrm{Ab}$ on guanine nucleotide-stimulated PDE activity in a biochemical assay using membranes from bovine rod outer segments (Liebman and Evanczuk, 1982; Malinski and Wensel, 1992). The membrane preparation was incubated with $2 \mathrm{mM} \mathrm{cGMP}$ and $0.1 \mathrm{~mm}$ GMPPNP, and PDE activity was monitored via changes of $\mathrm{pH}$. Low concentrations of $\mathrm{PIP}_{2}(1.8 \mu \mathrm{M})$ stimulated PDE activity by $\sim 50 \%$, and the stimulation decayed subsequently over $20 \mathrm{~min}$ (Fig. 11A). The PDE activity so stimulated could also be blocked by Zaprinast as expected (data not shown). Incubation of the assay mixture with $\mathrm{PIP}_{2} \mathrm{Ab}$ resulted in $80 \%$ inhibition of the PDE activity. Because the $\mathrm{PIP}_{2} \mathrm{Ab}$ preparation contains serum, we tested for effects of equivalent concentrations of FBS, and we tested for effects of a control Ab, IgG2b (Fig. 11B). FBS inhibited PDE activity, but the effect was very small compared with $\mathrm{PIP}_{2} \mathrm{Ab}$.

\section{DISCUSSION}

We have shown that PIP $_{2}$ has a strong inhibitory effect on rod CNG channels when $\alpha$ and $\beta$ subunits are expressed in Xenopus oocytes. Furthermore, effects on homomultimeric channels of $\alpha$ subunits are smaller and qualitatively different from effects on heteromeric channels with $\beta$ subunits. In the former case, $\mathrm{PIP}_{2}$ shifts the cyclic nucleotide dependence of channels to a higher 
concentration range; in the latter case, $\mathrm{PIP}_{2}$ produces a decrease in the apparent maximum current that can essentially eliminate current. Similar effects are observed in response to cytoplasmic MgATP, and those effects can be overcome by a PIP $_{2}$ antibody. Previously, tyrosine phosphorylation has been suggested to account for inhibitory effects of ATP on CNG channels expressed in oocyte membranes (Molokanova et al., 1997, 1999). Our work does not contradict that hypothesis, and one possibility is that inhibition by $\mathrm{PIP}_{2}$ and tyrosine phosphorylation are interdependent. In the ROMK potassium channels, for example, stimulatory effects of cAMP-dependent protein kinase phosphorylation and $\mathrm{PIP}_{2}$ binding are synergistic (Liou et al., 1999), and more detailed studies are required to test this possibility for inhibition of CNG channels.

The effects of $\mathrm{PIP}_{2}$ observed in amphibian rod cells are smaller in magnitude than effects observed with the $\alpha \beta$ heterotetramers in oocyte patches, but they are qualitatively similar in that maximal currents at high cGMP concentrations are reduced. We have tested several possible reasons for the difference in magnitude. First, amphibian rod CNG channels might respond differently than the cloned channels, which are bovine. However, we found very similar, small-magnitude $\mathrm{PIP}_{2}$ effects on cGMP-activated currents in patches from rod cells of mice (data not shown). Second, lipid phosphatases and phospholipases could be highly active in the rod cell membranes, so that $\mathrm{PIP}_{2}$ never accumulates. This seems unlikely because phosphatase inhibitors (orthovanadate and fluoride), Ca-free conditions, and a phospholipase $\mathrm{C}$ inhibitor (U73122) did not alter the $\mathrm{PIP}_{2}$ responses (data not shown). Third, lipids might not insert easily into the rod plasma membrane. However, we found that other phospholipids, such as PS and DAG (Fig. 9), did have significant effects. Also, we observed similar effects of $\mathrm{PIP}_{2}$ in patches from catfish cone cells in which disk membranes are contiguous with the plasma membrane (data not shown). Thus, the remaining possibilities are (1) a difference in the regulatory state of channels in oocytes and rods (e.g., phosphorylation by tyrosine kinases) and (2) a difference in accessory proteins that interact functionally with $\mathrm{CNG}$ channels in the oocyte versus rod membranes.

Our efforts to study similar reactions in rod patches have been complicated by the evidently very potent transphosphorylation reactions that result in PDE activation, even when using hydrolysis-resistant cGMP analogs. To account for our results, it seems essential to suggest that metabolism of phosphonucleotides is in some way compartmentalized under the rod outer segment membrane. The question is how. It is well established that guanylate kinase and NDK are present in rod outer segments (Berger et al., 1980; Hall and Kuhn, 1986), and NDK has been shown to interact with rod outer segments in a transducin-dependent manner (Orlov et al., 1997; Orlov and Kimura, 1998). It is thus not too surprising that the reaction sequence cIMP $\rightarrow$ IMP $\rightarrow$ IDP $\rightarrow$ ITP can be performed in patches without losing substrates to the bulk solution. What is more surprising is that pretreatment with cIMP and ATP can change the kinetics of GTP responses long after cIMP has been removed (Fig. 7). This suggests that nucleotide diphosphates and/or cyclic nucleotides can be sequestered in the vicinity of transducin and NDK for long periods of time. Furthermore, it suggests that the sequestered nucleotides can be metabolized and used preferentially by G-proteins when trinucleotides are infused from the bulk solution. One possibility is that nucleotide diphosphates remain bound to G-proteins during multiple phosphorylation-dephosphorylation cycles. A second possibility is that the nucleotide diphosphates dissociate to a neigh- boring binding site for rephosphorylation followed by rebinding. A third possibility is that noncatalytic cyclic nucleotide binding sites of PDE might provide a long-term "pool" of cyclic nucleotides for transphosphorylation reactions. Much further experimentation will be required to distinguish these possibilities. Regardless of the details, the enhancement of photoresponses in patches by ATP, described in Figure 7, suggests that transphosphorylation reactions are important determinants of G-protein activation in response to light.

As pointed out in Results, the inhibition of ATP responses by RGS9 supports the idea that ATP is ultimately acting through G-proteins. This result also suggests that the GTP hydrolysis rate of transducin can be enhanced many fold over its rate with a full complement of RGS9 present. Thus, RGS9 may be limiting for transducin inactivation kinetics, and there is clearly a kinetic potential to increase the G-protein kinetics by regulatory mechanisms.

Two final issues touched on by our experiments seem noteworthy. First, we have verified inhibitory effects of short-chain DAG on CNG channel current, but we have found no inhibitory effects of full-length DAG by two experimental means. Thus, we conclude that DAG probably does not physiologically inhibit CNG channel activity. Second, regarding possible $\mathrm{PIP}_{2}$ effects on the disk membranes, our major finding is that a $\mathrm{PIP}_{2}$ antibody can strongly inhibit PDE activity. This is suggestive of a regulatory role for $\mathrm{PIP}_{2}$ on PDE activity, but we did not obtain equivalent opposite effects of PIP, PIP $_{2}$ or PIP $_{3}$ in patches, nor did we obtain equivalent with other $\mathrm{PIP}_{2}$ ligands, such as neomycin (data not shown). Nevertheless, it is encouraging that $\mathrm{PIP}_{2}$ at low concentrations can indeed stimulate PDE activity in a biochemical assay (Fig. 11A). Clearly, further studies are required to test whether $\mathrm{PIP}_{2} \mathrm{Ab}$ is acting in a $\mathrm{PIP}_{2}$-specific manner in these experiments, and finally whether PIP $_{2}$ is a significant regulator of transducinPDE coupling.

\section{REFERENCES}

Abdulaev NG, Karaschuk GN, Ladner JE, Kakuev DL, Yakhyaev AV, Tordova M, Gaidarov IO, Popov VI, Fujiwara JH, Chinchilla D, Eisenstein E, Gilliland GL, Ridge KD (1998) Nucleoside diphosphate kinase from bovine retina: purification, subcellular localization, molecular cloning, and three-dimensional structure. Biochemistry 37:13958-13967.

Acharya JK, Janlink K, Hardy RW, Hartenstein V, Zuker CS (1997) InsP3 receptor is essential for growth and differentiation but not for vision in Drosophila. Neuron 18:881-887.

Berger SJ, DeVries GW, Carter JG, Schulz DW, Passonneau PN, Lowry OH, Ferrendelli JA (1980) The distribution of the components of the cyclic GMP cycle in retina. J Biol Chem 255:3128-3133.

Berman DM, Gilman AG (1998) Mammalian RGS proteins: barbarians at the gate [review]. J Biol Chem 273:1269-1272.

Chen CK, Wieland T, Simon MI (1996) RGS-r, a retinal specific RGS protein, binds an intermediate conformation of transducin and enhances recycling. Proc Natl Acad Sci USA 93:12885-12889.

Chen TY, Peng YW, Dhallan RS, Ahamed B, Reed RR, Yau KW (1993) A new subunit of the cyclic nucleotide-gated cation channel in retinal rods. Nature 362:764-767.

Choe HG, Ghalayini AJ, Anderson RE (1990) Phosphoinositide metabolism in frog rod outer segments. Exp Eye Res 51:167-176.

Chyb S, Raghu P, Hardie RC (1999) Polyunsaturated fatty acids activate the Drosophila light-sensitive channels TRP and TRPL. Nature 397:255-259.

Ertel EA (1990) Excised patches of plasma membrane from vertebrate rod outer segments retain a functional phototransduction enzymatic cascade. Proc Natl Acad Sci USA 87:4226-4230.

Ertel EA (1994) Modulation of guanylate cyclase and phosphodiesterase by monovalent cations and nucleoside triphosphates in light sensitive excised patches of outer rod segments. Pflügers Arch 428:372-381. 
Ghalayini AJ, Anderson RE (1992) Activation of bovine rod outer segment phospholipase C by arrestin. J Biol Chem 267:17977-17982.

Ghalayini AJ, Anderson RE (1995) Light adaptation of bovine retinas in situ stimulates phosphatidylinositol synthesis in rod outer segments in vitro. Curr Eye Res 14:1025-1029.

Gordon SE, Downing-Park J, Tam B, Zimmerman AL (1995) Diacylglycerol analogs inhibit the rod cGMP-gated channel by a phosphorylation-independent mechanism. Biophys J 69:409-417.

Hall SW, Kuhn H (1986) Purification and properties of guanylate kinase from bovine retinas and rod outer segments. Eur $\mathrm{J}$ Biochem 161:551-556.

Hayashi F, Amakawa T (1985) Light-mediated breakdown of phosphatidylinositol-4,5-bisphosphate in isolated rod outer segments of frog photoreceptor. Biochem Biophys Res Commun 128:954-959.

He W, Cowan CW, Wensel TG (1998) RGS9, a GTPase accelerator for phototransduction. Neuron 20:95-102.

Hilgemann DW, Ball R (1996) Regulation of cardiac $\mathrm{Na}^{+}, \mathrm{Ca}^{2+}$ exchange and KATP potassium channels by PIP2. Science 273:956-959.

Hilgemann DW, Lu CC (1998) Giant membrane patches: improvements and applications. Methods Enzymol 293:267-280.

Huang CL, Feng S, Hilgemann DW (1998) Direct activation of inward rectifier potassium channels by PIP2 and its stabilization by Gbetagamma. Nature 391:803-806.

Kaupp UB, Niidome T, Tanabe T, Terada S, Bonigk W, Stuhmer W, Cook NJ, Kangawa K, Matsuo H, Hirose T (1989) Primary structure and functional expression from complementary DNA of the rod photoreceptor cyclic GMP-gated channel. Nature 342:762-766.

Kelleher DJ, Dudycz LW, Wright GE, Johnson GL (1986) Ability of guanine nucleotide derivatives to bind and activate bovine transducin. Mol Pharmacol 30:603-608.

Klinker JF, Seifert R (1997) Functionally nonequivalent interactions of guanosine $5{ }^{\prime}$-triphosphate, inosine 5 -triphosphate, and xanthosine $5^{\prime}$ triphosphate with the retinal G-protein, transducin, and with Giproteins in HL-60 leukemia cell membranes. Biochem Pharmacol 54:551-562.

Liebman PA, Evanczuk AT (1982) Real time assay of rod disk membrane cGMP phosphodiesterase and its controller enzymes. Methods Enzymol 81:532-542.

Liou HH, Zhou SS, Huang C (1999) Regulation of ROMK1 channel by protein kinase A via a phosphatidylinositol 4,5-bisphosphatedependent mechanism. Proc Natl Acad Sci USA 96:5820-5825.

Malinski JA, Wensel TG (1992) Membrane stimulation of cGMP phosphodiesterase activation by transducin: comparison of phospholipid bilayers to rod outer segment membranes. Biochemistry 31:9502-9512.

Molokanova E, Trivedi B, Savchenko A, Kramer RH (1997) Modulation of rod photoreceptor cyclic nucleotide-gated channels by tyrosine phosphorylation. J Neurosci 17:9068-9076.

Molokanova E, Maddox F, Luetje CW, Kramer RH (1999) Activitydependent modulation of rod photoreceptor cyclic nucleotide-gated channels mediated by phosphorylation of a specific tyrosine residue. J Neurosci 19:4786-4795.

Orlov NY, Kimura N (1998) Interaction of nucleoside diphosphate kinase with membranes of bleached bovine retinal rod outer segments. Effects of $\mathrm{pH}$, salts, and guanine nucleotides. Biochemistry 63:171-179.

Orlov NY, Orlova TG, Reshetnyak YK, Burstein EA, Kimura N (1997) Interaction of recombinant rat nucleoside diphosphate kinase alpha with bleached bovine retinal rod outer segment membranes: a possible mode of $\mathrm{pH}$ and salt effects. Biochem Mol Biol Int 41:189-198.

Otero AD (1990) Transphosphorylation and G protein activation [review]. Biochem Pharmacol 39:1399-1404.

Papermaster DS, Dreyer WJ (1974) Rhodopsin content in the outer segment membranes of bovine and frog retinal rods. Biochemistry 13:2438-2444.

Peng YW, Rhee SG, Yu WP, Ho YK, Schoen T, Chader GJ, Yau KW (1997) Identification of components of a phosphoinositide signaling pathway in retinal rod outer segments. Proc Natl Acad Sci USA 94:1995-2000.

Piacentini L, Niroomand F (1996) Phosphotransfer reactions as a means of $\mathrm{G}$ protein activation [review]. Mol Cell Biochem 157:59-63.

Ranganathan R, Malicki DM, Zuker CS (1995) Signal transduction in Drosophila photoreceptors [review]. Annu Rev Neurosci 18:283-317.

Swarup G, Garbers DL (1983) Stimulation of rhodopsin phosphorylation by guanine nucleotides in rod outer segments. Biochemistry 22:1102-1106.

Tanaka JC, Eccleston JF, Furman RE (1989) Photoreceptor channel activation by nucleotide derivatives. Biochemistry 28:2776-2784.

Van Rooijen LA, Bazan NG (1986) The inositide cycle in bovine photoreceptor membranes. Life Sci 38:1685-1693.

Varnum MD, Black KD, Zagotta WN (1995) Molecular mechanism for ligand discrimination of cyclic nucleotide-gated channels. Neuron 15:619-625.

Yan H, Tsai MD (1999) Nucleoside monophosphate kinases: structure, mechanism, and substrate specificity [review]. Adv Enzymol Relat Areas Mol Biol 73:103-134.

Yee R, Liebman PA (1978) Light-activated phosphodiesterase of the rod outer segment. Kinetics and parameters of activation and deactivation. J Biol Chem 253:8902-8909.

Yuen PST, Graeff RM, Walseth TF, Goldberg ND (1989) Non-identity of cGMP as the guanine nucleotide stimulated to bind to ROS by light and ATP. Exp Eye Res 49:75-85. 